\title{
ARTICLE OPEN Ibrutinib as a potential therapeutic for cocaine use disorder
}

\author{
Spencer B. Huggett $\mathbb{D}^{1 \times}{ }^{凶}$, Jeffrey S. Hatfield $\mathbb{D}^{2}$, Joshua D. Walters ${ }^{2}$, John E. McGeary ${ }^{3,4}$, Justine W. Welsh (iD) ${ }^{5}$, Trudy F. C. Mackay ${ }^{2}$,
} Robert R. H. Anholt ${ }^{2}$ and Rohan H. C. Palmer ${ }^{1 \otimes}$

This is a U.S. government work and not under copyright protection in the U.S.; foreign copyright protection may apply 2021

Cocaine use presents a worldwide public health problem with high socioeconomic cost. No current pharmacologic treatments are available for cocaine use disorder (CUD) or cocaine toxicity. To explore pharmaceutical treatments for tthis disorder and its sequelae we analyzed gene expression data from post-mortem brain tissue of individuals with CUD who died from cocaine-related causes with matched cocaine-free controls ( $n=71, M_{\text {age }}=39.9,100 \%$ male, 49\% with CUD, 3 samples/brain regions). To match molecular signatures from brain pathology with potential therapeutics, we leveraged the L1000 database honing in on neuronal mRNA profiles of 825 repurposable compounds (e.g., FDA approved). We identified 16 compounds that were negatively associated with CUD gene expression patterns across all brain regions $\left(p_{\text {adj }}<0.05\right)$, all of which outperformed current targets undergoing clinical trials for CUD (all $p_{\text {adj }}>0.05$ ). An additional 43 compounds were positively associated with CUD expression. We performed an in silico follow-up potential therapeutics using independent transcriptome-wide in vitro (neuronal cocaine exposure; $n=18$ ) and in vivo (mouse cocaine self-administration; $n=12-15$ ) datasets to prioritize candidates for experimental validation. Among these medications, ibrutinib was consistently linked with the molecular profiles of both neuronal cocaine exposure and mouse cocaine self-administration. We assessed the therapeutic efficacy of ibrutinib using the Drosophila melanogaster model. Ibrutinib reduced cocaine-induced startle response and cocaine-induced seizures ( $n=61-142$ per group; sex: $51 \%$ female), despite increasing cocaine consumption. Our results suggest that ibrutinib could be used for the treatment of cocaine use disorder.

Translational Psychiatry (2021)11:623; https://doi.org/10.1038/s41398-021-01737-5

\section{INTRODUCTION}

To date, there are no medications approved by the Food and Drug Administration (FDA) for human CUD or cocaine toxicity. Approximately 18.1 million people use cocaine globally each year [1] and roughly $30 \%$ of those users reside in the United States [2] (US). In the US, nearly 1 million individuals meet criteria for cocaine use disorder [2] (CUD) and 13\% of the individuals in North American substance use treatment facilities are treated for CUD [1]. Cocaine use increases risk for cardiovascular disease, seizures, and mental health disorders and contributes to roughly $40 \%$ of drug-related emergency room visits in the US [3]. Cocaine exerts adverse neurological effects in the brain, and at high doses can lead to cardiovascular disease and death. Since 1999, cocaineinvolved overdose deaths have increased by $416 \%$ in the US and resulted in 15,883 deaths in 2019 [4].

While behavioral treatments for CUD exist, they have limited efficacy and are plagued by high dropout rates [5]. Over 20 pharmcologic agents are currently being tested for clinical trials for CUD. Most of these medications include antidepressants, antipsychotics, psychostimulants, cognitive enhancing drugs, anxiolytics, repurposed medications for other substance use disorders, anticonvulsants/muscle relaxants, and dopamine agonists [6]. These treatments generally have mixed efficacy and often only demonstrate effects in particular subpopulations.
Medications for cocaine toxicity are also limited. Emergency room practitioners rely on beta-blockers for cocaine-induced cardiotoxicity [7] and benzodiazapines for cocaine-induced seizures [8]. Many candidate compounds for treating CUD and cocaine toxicity rely on repurposing existing medications, leveraging knowledge of cocaine's pharmacology, and/or the biological mechanisms underpinning cocaine pathology. For instance, therapeutics that enhance cocaine metabolism (cocaine esterases) have been proposed to reverse cocaine overdose [9].

The molecular brain pathology of CUD is characterized by persistent cellular and molecular adaptations across multiple brain regions, particularly in the mesocorticolimbic "reward" pathway. Molecular adaptations in this brain pathway mediate reward, motivation, behavioral control, memory formation, incentive salience, cue, drug and stress-induced drug taking/relapse. One approach to investigate the molecular brain correlates underlying CUD (and potentially cocaine toxicity) is to examine gene expression profiles in post-mortem brain tissue by comparing individuals with CUD to matched cocaine-free controls. Currently, three studies are publicly available that assess CUD gene expression profiles in brain regions associated with addiction: the dorsal-lateral prefrontal cortex [10] (dIPFC), hippocampus [11] and midbrain [12]. Previously, medications that consistently target gene expression across meso-cortico-limbic brain regions have

\footnotetext{
${ }^{1}$ Behavioral Genetics of Addiction Laboratory, Department of Psychology at Emory University, Atlanta, GA, USA. ${ }^{2}$ Department of Genetics and Biochemistry and Center for Human Genetics, Clemson University, Greenwood, SC, USA. ${ }^{3}$ Department of Psychiatry and Human Behavior, Brown University, Providence, RI, USA. ${ }^{4}$ Providence Veterans Affairs Medical Center, Providence, RI, USA. ${ }^{5}$ Department of Psychiatry and Behavioral Sciences, Emory University School of Medicine, Atlanta, GA, USA. ${ }^{\circledR}$ email: Spencer.B.Huggett@emory.edu; Rohan.Palmer@Emory.edu
}

Received: 11 May 2021 Revised: 21 October 2021 Accepted: 1 November 2021

Published online: 08 December 2021 


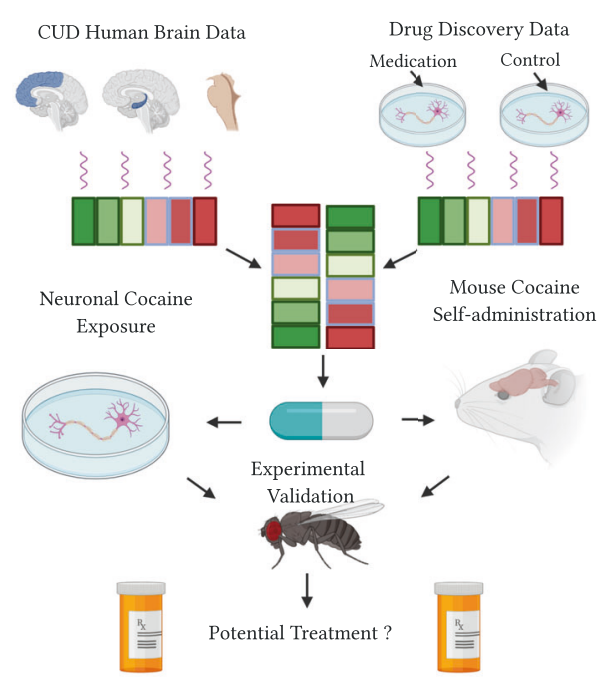

Fig. 1 Pictoral overview of the study. This schematic outlines the stages of the study from drug discovery (top), to computational follow-up (middle) and in-vivo validation (bottom).

demonstrated robust effects for reducing binge drinking in mice [13]. Thus, screening medications that target transcriptional patterns across relevant brain regions may help identify FDAapproved therapeutics that could be repurposed for treating CUD.

Drug-discovery analyses can cut costs and time for developing effective therapeutics, which on average cost $\$ 1.4$ billion and take 12-16 years to develop [14]. The NIH-funded Library of Integrated Network-based Cellular Signatures (LINCS L1000) database facilitates drug development strategies by indexing over 1.3 million expression profiles resulting from an extensive catalog of more than 48,000 perturbagens (pharmaceuticals, small molecules, shRNAs, cDNAs, and biologicals) in over 80 human cell lines [15]. This resource allows rapid screening of compounds that may target the molecular mechanisms of a disease.

Here, we outline a neuronal mRNA drug-repurposing framework that identifies and validates potential medications for CUD and/or cocaine toxicity (see Fig. 1 for study overview).

\section{METHODS \\ Drug-discovery input}

Our human sample $(n=71)$ used publicly available gene expression data from post-mortem brain tissue of individuals with cocaine use disorder (CUD; $n=36)$ and matched cocaine-free controls $(n=35)$. Data were obtained from three independent studies (GSM2642566 [10]; SRA029279 [11]; E-GEOD-54839 [12]; Supplementary Table S1).

We conducted drug discovery analyses leveraging the L1000 database, which catalogues mRNA expression profiles of human cell types exposed to therapeutic compounds [15]. The input for our drug discovery analysis included three categories. The first category included all differentially expressed genes (Benjamini-Hochberg False Discovery Rate $(F D R)<0.05$ ) associated with CUD in the dIPFC [16], hippocampus [17] and midbrain [12]. Differentially expressed genes might include genes attributed to cocaineinduced toxicity, cardiac complications or psychiatric comorbidities. Thus, our second category focused on the genes related to the behavioral manifestations of CUD, by including the mRNA expression of genes from the cocaine addiction pathway (Kyoto Encyclopedia of Genes and Genomes [KEGG] database). The last category sought to further minimize potential biases of measurement error by employing "landmark" genes (those directly measured from L1000, rather than imputed) that were part of at least one of the first two categories. We refer to the input genes for our drug discovery analysis as CUD genes (Supplementary File S1).

\section{Drug-discovery analyses}

We used a signature matching technique to find potential therapeutics for CUD or cocaine toxicity. Our analyses utilized the L1000 Connectivity
Mapping gene expression profiles from the Library of Integrated Network Based Cellular Signatures (level 5 data from phase I: GSE92742 and phase II: GSE70138). Since the focus of the study was to find repurposable treatments for CUD, we selected all compounds that were FDA approved or in stages 1-3 of clinical trials - as listed from the drug-repurposing hub website (https://clue.io/repurposing\#download-data; updated 3/24/2020). We benchmarked our findings with treatments that were currently undergoing clinical trials for CUD and specifically those that were reported on the https://clinicaltrials.gov/ website.

We focused our drug discovery analysis on two neuronal cell types used in the L1000 database: differentiated neuronal cells and neuronal progenitor cells. In total, we evaluated the potential therapeutic value of 825 compounds, which spanned 3468 individual neuronal mRNA signatures (in vitro gene expression profiles for a compound measured at a particular dose, time, and cell line). For each signature, we estimated a linear Pearson Product-Moment correlation coefficient with the CUD input. Note that not all medications undergoing clinical trials for CUD were included in the human neuronal cell lines from the L1000 database (Supplementary File S2).

To identify potential treatments for CUD, we conducted multi-level meta-regressions for all 825 compounds using the metafor package in $\mathrm{R}$ [18]. Multi-level meta-regression provides a powerful and interpretable framework that can accommodate complex data types. Our metaregressions adjusted for two random effects: human brain region (or study) and the three input categories within studies (e.g. KEGG $\mathrm{ClPFC}_{\text {, }}$ $\mathrm{KEGG}_{\text {Hippocampus }}$ etc.). We treated each compound as a fixed-effect incorporating the effect size $(r)$ and sampling variability $\left(s e^{2}{ }_{r}\right)$ for neuronal signatures of a compound. Theoretically, if the transcriptional signature of a compound is negatively associated with a disease, this compound may 'reverse' the underlying disease mechanisms and may increase the likelihood of demonstrating clinical utility. Hence, we emphasized compounds with negative meta-regression coefficients that also survived correction for multiple testing (FDR $<0.05)$ and defined these compounds as potential therapeutics. As medications with positive associations may also exert a clinical benefit, we also explored medications that were positively associated with CUD and report on these in the supplement.

We used a computational follow-up approach to prioritize the potential therapeutics identified from our drug discovery analysis using two independent transcriptome-wide datasets. We queried gene expression studies via GEO on March 2020 to find gold standard preclinical models of rodent self-administration datasets that matched brain regions and cell types relevant to the human post-mortem data. The datasets used in our study included a human neuronal cocaine exposure model [19] (SH-SY5Y neuroblastoma cells' GSE71939) and a mouse model of cocaine selfadministration [20] (GSE110344). The neuronal cocaine exposure dataset included 18 samples that assessed mRNA expression (via microarray) for two time points ( 6 or $24 \mathrm{~h}$ post exposure) across three doses $(0 \mu \mathrm{M}, 1 \mu \mathrm{M}$ and $5 \mu \mathrm{M}$; the latter two mimic clinically relevant cocaine levels found in individuals with cocaine abuse). The mouse RNA-sequencing (RNA-seq) data were collected from 12-15 male C57BL/6J mice using similar brain regions to the human samples (ventral tegmental area (VTA), hippocampus and PFC). Mice pressed a lever to receive intravenous infusions of cocaine ( $1 \mathrm{mg} / \mathrm{kg} ; 2 \mathrm{~h}$ sessions for 2 weeks) or saline. Brain tissue was extracted $24 \mathrm{~h}$ after the last self-administration session and mouse modeling was limited to orthologous genes listed from the mouse genome informatics dataset (http://www.informatics.jax.org/).

To maintain consistency, we harmonized the data processing and analyses for our validation datasets to resemble the human brain data (see Supplementary Methods). Using multi-level meta-regression, we assessed whether the potential CUD treatments were also negatively associated with the differential expression of the CUD genes in the neuronal cocaine exposure and mouse cocaine self-administration datasets-accounting for dose and time (neuronal exposure data) or brain region (mouse data) as random effects. Results from the computational follow-up were used to prioritize medications for experimental validation.

\section{Behavioral assays}

Drosophila melanogaster (Canton-S B strain) were reared on cornmealmolasses-yeast medium at $25^{\circ} \mathrm{C}$ and $70 \%$ humidity under a $12 \mathrm{~h}$ light-dark cycle (lights on at 6:00 am). Cocaine-HCl was obtained from the National Institute on Drug Abuse under Drug Enforcement Administration license RA0443159. Flies were food-deprived for $24 \mathrm{~h}$ followed by a $20 \mathrm{~m}$ freefeeding period. During this period, flies consumed one of four food formulations containing either no treatment $(n=66$ females, $n=61$ males), cocaine only ( $n=141$ females, $n=140$ males), cocaine and 
A

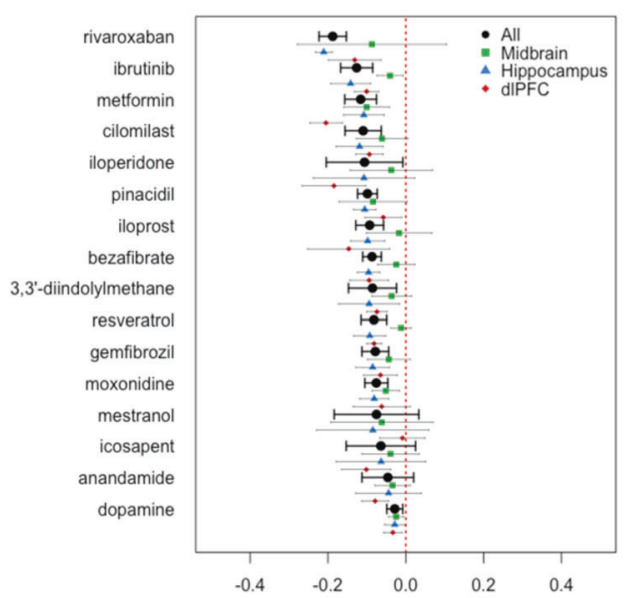

B

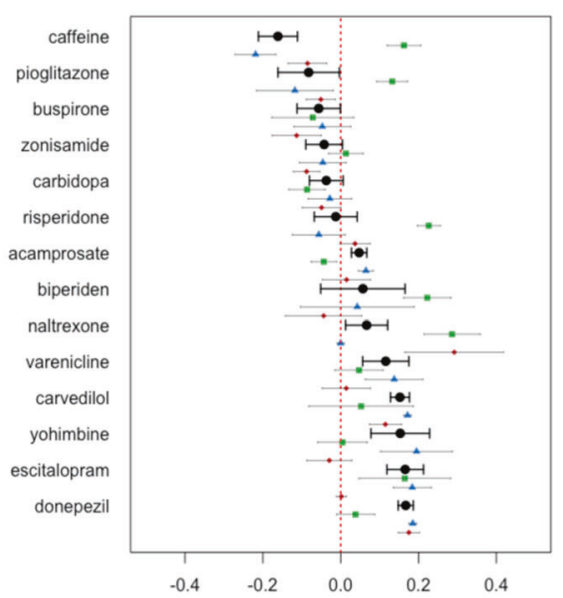

Drug Discovery Compound_Disease Associations ( Mean $_{\text {Weighted }} \mathrm{r}$ )

Fig. 2 Drug-discovery results for CUD using human brain data. Panel (A) shows repurposable compounds negatively associated with CUD gene expression in the brain (all $p_{\text {adj }}<0.05$ ). Panel (B) shows the results of the medications currently undergoing clinical trials for CUD. The $x$ axis represents the weighted average of a compound's correlation coefficients with the human brain CUD genes and each compound's weighted standard error bars. Also, note that the black circles represent the effect across all brain regions/studies and the points below are color coded by brain region.

ibrutinib ( $n=142$ females, $n=132$ males) or ibrutinib only ( $n=68$ females, $n=66$ males). For all conditions, we observed no significant differences between ibrutinib treated flies and flies undergoing no treatment (all $t=$ 0.37994 , all $p>0.704$ ). Thus, we reported and visualized the differences between the other three groups. The concentrations of cocaine- $\mathrm{HCl}$ and ibrutinib (Tocris Bioscience; Bristol, UK; Product No: 6813; Batch No: 2A/ $247900)$ were both $0.16 \%(\mathrm{w} / \mathrm{w})$. The concentrations of cocaine used were based on optimal concentrations that give rise to phenotypic effects without causing lethality determined previously [21].

We quantified cocaine consumption in the Drosophila melanogaster model. All food formulations included 1\% (w/w) FD\&C Blue \#1 dye. Following behavioral testing for each fly, dye was extracted into $300 \mu \mathrm{L}$ of deionized $\mathrm{H}_{2} \mathrm{O}$ using Fisher brand Bead Mill 4 (Speed: $4 \mathrm{~m} / \mathrm{s}$; Time: $15 \mathrm{sec}$ ). To settle fly debris, tubes were centrifuged at $13,000 \times R C F$ for 1 min using an Eppendorf Centrifuge 5430R. To quantify consumption, $100 \mu \mathrm{L}$ of each extract was dispensed in duplicate into wells of a 384-well microplate. Absorbance was measured at $630 \mathrm{~nm}$ using a SpectraMax iD5 Microplate Reader. Dye concentrations were calculated using a standard curve (FD\&C Blue \#1 in deionized $\mathrm{H} 2 \mathrm{O} ; 0.0-6.0 \mu \mathrm{g} / \mathrm{mL}$ ).

Immediately following exposure, each fly underwent behavioral testing for startle response induced by a mechanical disturbance. A vial housing a single fly was dropped through a chute from a height of $42 \mathrm{~cm}$ and then secured in a horizontal position. During the next $45 \mathrm{~s}$, the total time spent moving and the occurrence of seizures were recorded. Seizure activity was defined as significant disruption of normal movement with severe tremors and muscle twitching (see Supplementary File S3). While immobile, seizing, or grooming, flies were considered stationary. Startle response data were analyzed using a two-way fixed effects factorial ANOVA model: $Y=M+S$ $+T+S^{*} T+\varepsilon$, where $Y$ is the phenotype, $M$ is the mean, $S$ is sex, $T$ is treatment, $S{ }^{*} T$ is the sex by treatment interaction, and $\varepsilon$ is the residual. We used the type III Sums of Squares due to differing sample sizes across groups. Post-hoc comparisons were performed using Student's $t$ tests. Seizure data were analyzed using Fisher's exact tests in R.

\section{RESULTS}

\section{Drug-discovery input}

A schematic overview of the study design is presented in Fig. 1. Prior to our drug discovery analysis we examined the input data. We observed minimal overlap among differentially expressed genes identified across human brain regions (see Supplementary File S1). Associations among KEGG cocaine addiction genes were modest and sometimes negative $\left(r_{\text {midbrain_hippocampus }}=-0.31, p=\right.$ $0.044 ; r_{\text {dIPFC_hippocampus }}=0.02, p=0.915 ;$ and $r_{\text {midbrain_dIPFC }}=0.36$, $p=0.019)$. This heterogeneity and individual variation may be one factor contributing to the difficulty in finding an effective treatment that targets multiple brain regions and is effective for all individuals in a population.

\section{Drug-discovery analyses}

Our drug discovery analysis discovered 16 medications with gene expression signatures that were negatively associated with gene expression associated with CUD in the midbrain, hippocampus and dIPFC ( $p_{\text {adj }}<0.05$; Fig. 2A). These medications had diverse pharmacological mechanisms of action (Supplementary Table S2) and outperformed the current pharmaceuticals undergoing clinical trials for CUD (Fig. 2B). We also show the medications that were positively associated with CUD in the supplement (see Supplementary Fig. S2; see Supplementary File S4 for all results).

Next, we employed a computational follow-up of the 16 potential CUD medications using transcriptome-wide data from preclinical in vitro and in vivo models. Only one compound, ibrutinib, was negatively associated with neuronal cocaine exposure $\left(M_{r}=-0.017, \mathrm{se}_{r}=0.006, p=0.001 ; p_{\text {adj }}=0.019\right)$ and was trending to be negatively associated with the gene expression profiles associated with mouse cocaine self-administration $\left(M_{r}=\right.$ $-0.017, \mathrm{se}_{r}=0.007, p=0.007 ; p_{\mathrm{adj}}=0.0732$; Fig. 3). No other medication that was negatively associated with CUD expression demonstrated significant evidence among preclinical models (see Supplementary Fig. S3). Using correlations between human CUD brain data and the in vitro drug discovery data, we found negative associations between ibrutinib and the expression of genes involved in dopaminergic and glutamatergic neurotransmission as well as various immediate early genes, intracellular signaling cascade genes and putative neuroepigenetic transcripts (Fig. 4).

\section{Validation of potential therapeutic efficacy of ibrutinib in the Drosophila model}

To validate the effectiveness of ibrutinib as a potential therapeutic for CUD, we evaluated its effects on cocaine-induced phenotypes in the Drosophila melanogaster model. Drosophila provides an advantageous model system for studies on cocaine consumption [22]. The Drosophila dopamine transporter contains a binding site that can accommodate cocaine [23], and exposure to cocaine gives rise to motor responses that resemble behaviors observed in 
rodents. In addition, flies develop sensitization to repeated intermittent exposure to cocaine [24, 25].

We measured cocaine consumption, startle behavior and the prevalence of seizure activity in male and female flies following acute consumption of solid food, solid food supplemented with cocaine, or solid food supplemented with cocaine and ibrutinib. Male flies exposed to cocaine spent less time moving after being subjected to a mechanical startle (Fig. 5A). This is likely due to the occurrence of cocaine-induced seizures, which were scored as

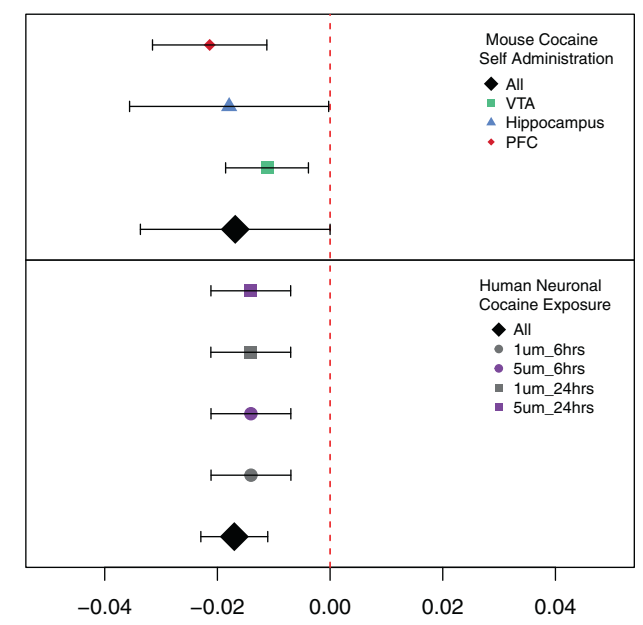

Drug Discovery Compound_Cocaine Associations ( Mean $_{\text {Weighted }} \mathrm{r}$ )

Fig. 3 Computational follow-up in preclinical models of cocaine use. The top panel shows associations between the neuronal signatures of ibrutinib and differential expression results from the in vitro cocaine neuronal exposure data. The bottom panel displays the associations between the neuronal signatures of ibrutinib with differential expressed genes from the mouse cocaine selfadministration dataset. stationary periods. Both male and female flies that consumed ibrutinib with cocaine spent more time moving than flies that only consumed cocaine (Fig. 5A). Male flies that consumed ibrutinib and cocaine showed a significant decrease in the prevalence of seizures (Fig. 5B). Fewer cocaine-induced seizures were also observed in lbrutinib treated females, but this observation did not reach statistical significance since the incidence of cocaineinduced seizures was lower in females than in males. To ascertain that ibrutinib did not reduce food intake, we measured cocaine consumption with food supplemented with ibrutinib alone and found that ibrutinib increased cocaine consumption in male flies and was trending to do so in female flies (see Fig. 5C; see Supplementary File S5 for data). Altogether, these results indicate that ibrutinib may be useful as a therapeutic to prevent various neurobehavioral and toxic effects of cocaine use.

\section{DISCUSSION}

We identified potentially repurposable medications for CUD and/ or cocaine toxicity, which demonstrated more reproducible associations across the brain's reward circuitry than current medications undergoing clinical trials in the US. Despite the low correspondence of gene expression across datasets, ibrutinib was consistently negatively associated with patterns of gene expression observed in brains of individuals with CUD across brain regions and diverse samples as well as across two gold standard preclinical models. Ibrutinib decreased cocaine-induced seizures in the Drosophila model and thus is a promising repurposable medication for cocaine-induced seizures and possibly for other aspects of CUD that cannot readily be evaluated in Drosophila. We did not attempt to validate medications positively associated with CUD (see Supplementary Fig. S2).

Ibrutinib is an irreversible Bruton's tyrosine kinase (BTK) inhibitor and is approved by the FDA for various $B$ cell cancers including chronic lymphocytic leukemia [26]. BTK inhibitors have demonstrated efficacy for multiple sclerosis [27]—via an antiinflammatory mechanism - and have also reduced binge drinking
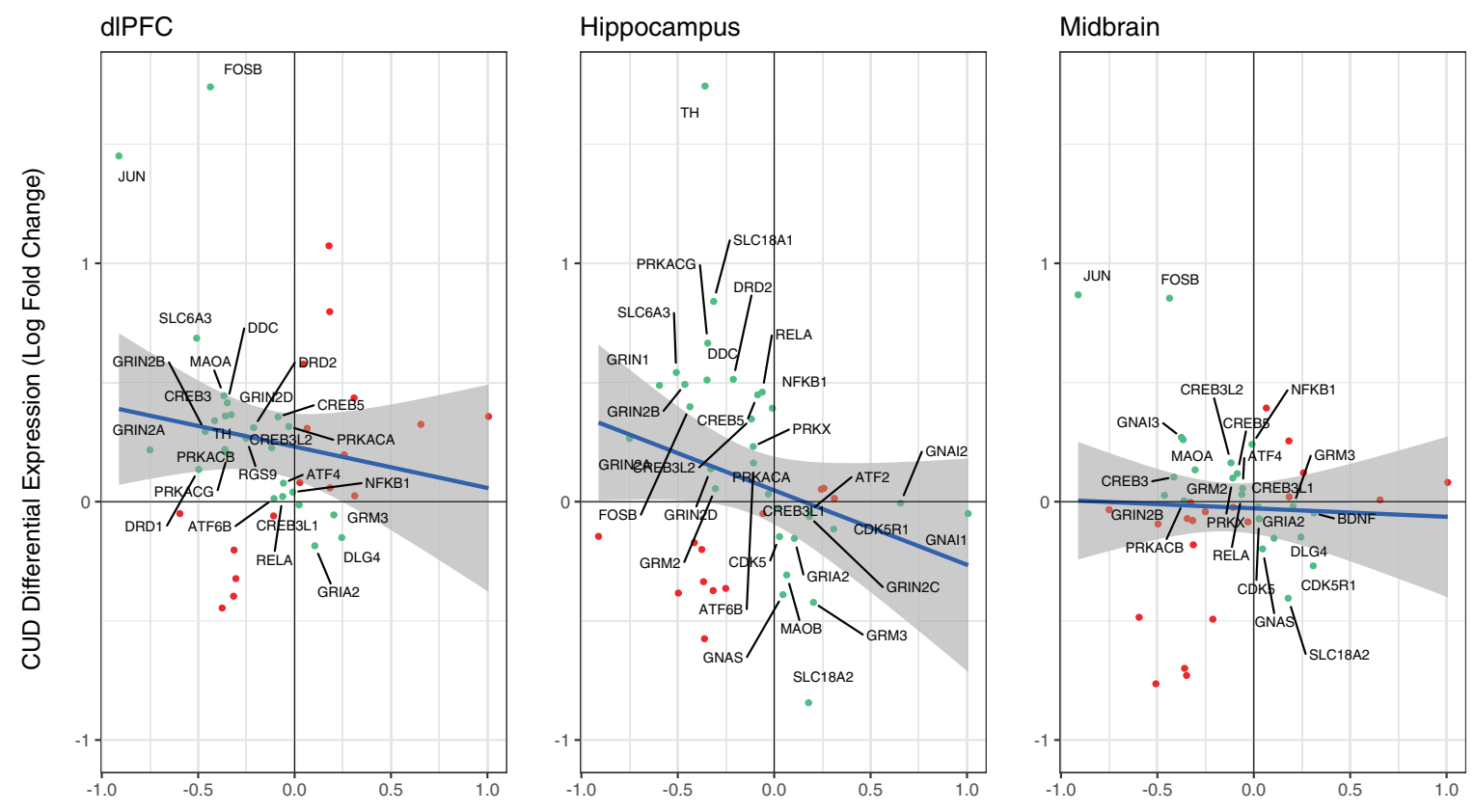

Ibrutinib Differential Expression (L1000: Mean Z-score Across Neuronal Signatures)

Fig. 4 Neuronal signatures of ibrutinib are negatively associated with cocaine addiction genes in the human brain. The blue line shows the best fitting line and standard error between CUD gene expression (of KEGG Cocaine Addiction Pathway genes) and neuronal signatures of ibrutinib (collapsing across dose, time and cell line; $r_{\text {dIPFC }}=-0.16, r_{\text {hippocampus }}=-0.25$ and $r_{\text {midbrain }}=-0.04$ ). Genes were labeled if they were in the negative diagonal of the plot (green points). Differential expression of FOSB in the dIPFC was windsorized for visualization purposes. 
A

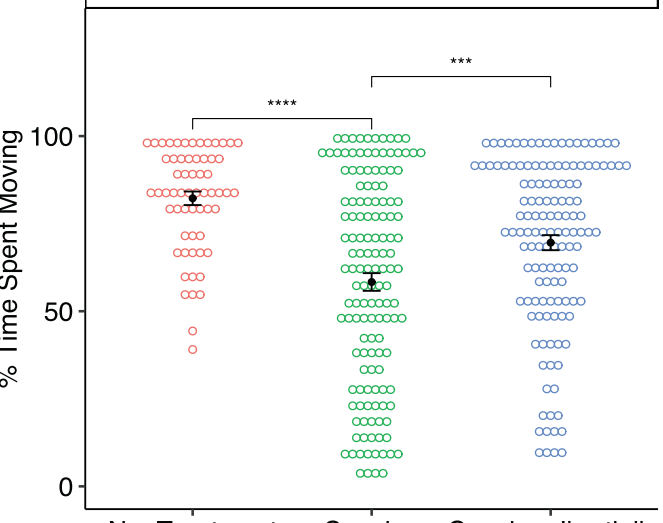

Female

No_Treatment Cocaine Cocaine_Ibrutinib No_Treatment Cocaine Cocaine_Ibrutinib Treatment
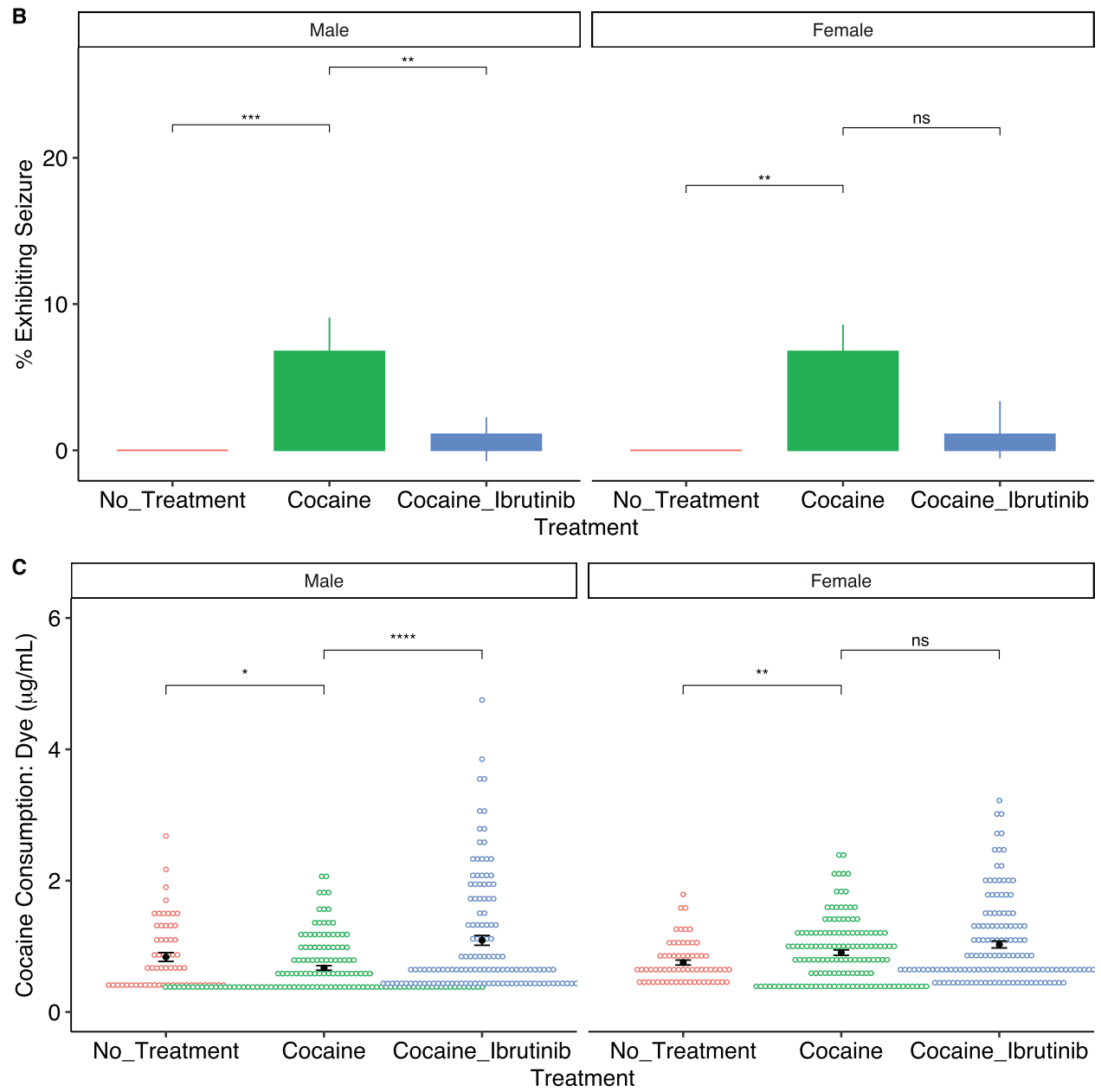

Fig. 5 Ibrutinib influences cocaine-induced startle response, cocaine-induced seizures and cocaine consumption in Drosophila

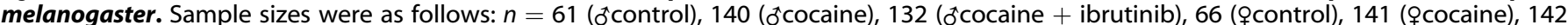
(ococaine + ibrutinib). A Dotplot showing the cocaine startle response by condition. B Barplot showing the average seizure activity during the startle response across conditions with $95 \%$ confidence intervals. C Dotplot showing cocaine consumption across conditions. Colored dots represent individual flies and the black dot displays the average for each group and the standard error.ns $p>0.05$. ${ }^{*} p<0.05$. ${ }^{* *} p<0.01$. ${ }^{* * *} p<0.001{ }^{* * * *} p<0.001$.

in mice [13]. BTKs are predominantly expressed in microglia in the brain (https://www.brainrnaseq.org/) and amplify intracellular signals from (B cell) receptors to the nucleus [28]. Cocaine activates overlapping intracellular signaling pathways with canonical B cell signaling pathways (e.g., JUN, RELA, and NFKB1 genes) and is associated with a pro-inflammatory response in the brain
[29]. We postulate that inhibiting BTK via ibrutinib interferes with the cocaine response via disruption of intracellular signaling cascades and/or evoking anti-inflammatory processes.

In addition to ibrutinib, we discovered other potential medications that may correlate with brain profiles of CUD. Some of these compounds may target molecular processes underlying chronic 
cocaine use. For instance, we found that dopamine was negatively associated with the differential expression of CUD genes across brain regions, which may counteract the hypodopaminergic state induced by chronic cocaine use [30]. Various drugs identified by our analyses have been previously used to treat cardiac complications, such as Rivaroxaban, Pinacidil, Iloprost, Gemfibrozil, Bexafibrate and Moxonidine. It is possible that these medications exert a protective role for the cardiovascular consequences of chronic cocaine use. Some of these medications may have both health and behavioral benefits. For instance, Moxonidine reduces (cueinduced) cocaine relapse [31] and ethanol withdrawal [32, 33] in rodents. Of note, the pharmacological profiles of the potential CUD medications we identified have shown to reduce various drug use behaviors (PPAR alpha agonists [34]; PDE4 inhibitors [35, 36]). Potential CUD medications also influenced catecholamine neurotransmission, potassium channels, hormonal signaling and mitochondrial processes, which have all been implicated in the pathophysiology of cocaine use [37-40]. Our approach may serve a dual purpose for indirectly unraveling molecular brain features of human CUD while also identifying specific chemical compounds that may demonstrate clinical utility for cocaine-related outcomes.

The results of our study should be interpreted with caution until further validation studies are conducted in different species and behavioral paradigms. We used all extant transcriptome-wide brain data on human CUD, but these sample sizes were small, ascertained from highly selected male cocaine users and generally included heterogenous cell types in the brain. These data are unable to differentiate specific symptoms of CUD and may have bias relating to health and psychiatric comorbidities. Whereas the Drosophila model can evaluate some behavioral effects following acute consumption of cocaine, many aspects of cocaine use, addiction, withdrawal, and death cannot be assessed in the fly model, but require complementary studies in other model systems. Future studies in mammalian models are warranted to determine the efficacy, dose, and safety of ibrutinib for cocaine-related outcomes. The L1000 drug discovery database assesses acute gene expression profiles of medications in human cell lines and is thus limited in understanding chronic therapeutic administration and in vivo mechanisms of medications. The lead repurposable drug from this study, ibrutinib, may not be an accessible treatment option to many, as it can be costly and while it is generally well tolerated it can have various side effects (e.g., diarrhea, infection and fatigue) [41] and off target effects (arrhythmias, platelet dysfunction, QTc prolongation) that may limit its utility for cocainerelated disease indications. Additionally, it is unknown what dose would be most effective and whether negative health consequences arise when combining this medication with cocaine (or crack cocaine). Despite these limitations, ibrutinib emerges as a promising therapeutic with potential, at least for the treatment of cocaine-induced seizures. In a broader context, our study provides a proof of principle for a powerful, flexible and interpretable strategy that can be used to identify and prioritize therapeutics from genome-wide and transcriptome-wide datasets.

\section{REFERENCES}

1. United Nations Publication SNE 19. X 8. World drug report. United Nations Publication; 2019.

2. SAMSHA. Key Substance Use and Mental Health Indicators in the United States: Results from the 2018 National Survey on Drug Use and Health. 2019.

3. Turner C, Chandrakumar D, Rowe C, Santos G, Riley D, Coffin PO. Cross-sectional cause of death comparisons for stimulant and opioid mortality in San Francisco, 2005-2015. Drug Alcohol Depend. 2019;185:305-12.

4. National Institute on Drug Abuse [Internet]. Trends \& Statistics: Overdose death rates [updated Jan 29 2021; cited Feburary 5, 2021]. Available from: https://www. drugabuse.gov/drug-topics/trends-statistics/overdose-death-rates.

5. Dutra L, Stathopoulou G, Basden SL, Leyro TM, Powers MB, Otto MW. A metaanalytic review of pyschosocial interventions for substance use disorders. Am J Pyschiatry. 2006;14:3989-96.
6. Chan B, Kondo K, Freeman M, Ayers C, Montgomery J, Kansagara D. Pharmacotherapy for cocaine use disorder-a systematic review and meta-analysis. J Gen Intern Med. 2017;24:2858-2873.

7. Vroegop MP, Franssen EJ, Voort PHJ, van den Berg TNA, Langewag RJ, Kramers C. The emergency care of cocaine intoxications. Neth J Med. 2009;67:122-126.

8. Chen $\mathrm{H}$, Albertson TE, Olson KR. Treatment of drug-induced seizures. Br J Clin Pharm. 2015;81:412-9.

9. Narasimhan D, Woods JH, Sunahara RK. Bacterial cocaine esterase: a proteinbased therapy for cocaine overdose and addiction. Future Med Chem. 2012;4:137-50.

10. Ribeiro EA, Scarpa JR, Garamszegi SP, Kasarskis A, Mash DC, Nestler EJ. Gene network dysregulation in dorsolateral prefrontal cortex neurons of humans with cocaine use disorder. Sci Rep. 2017;7:1-10.

11. Zhou Z, Yuan Q, Mash DC, Goldman D. Substance-specific and shared transcription and epigenetic changes in the human hippocampus chronically exposed to cocaine and alcohol. Proc Natl Acad Sci. 2011;108:6626

12. Bannon MJ, Johnson MM, Michelhaugh SK, Hartley ZJ, Halter SD, David JA, et al. A molecular profile of cocaine abuse includes the differential expression of genes that regulate transcription, chromatin, and dopamine cell phenotype. Neuropsychopharmacology. 2014;39:2191-9.

13. Ferguson LB, Ozburn AR, Ponomarev I, Metten P, Reilly $M$, Crabbe JC, et al. Genome-wide expression profiles drive discovery of novel compounds that reduce binge drinking in mice. Neuropsychopharmacology. 2018;43:1257-66.

14. Nosengo N. New tricks for old drugs. Nature 2016;534:314-6.

15. Subramanian A, Narayan R, Corsello SM, Root DE, Wong B, Golub TR, et al. A next generation connectivity map: L1000 platform resource. Cell. 2017;171:1437-e17.

16. Huggett SB, Stallings MC. Genetic architecture and molecular neuropathology of human cocaine addiction. J Neurosci. 2020;40:5300-13.

17. Huggett SB, Stallings MC. Cocaine'omics: Genome-wide and transcriptome-wide analyses provide biological insight into cocaine use and dependence. Addict Biol. 2019;25:1-10.

18. Viechtbauer W. Conducting meta-analyses in $\mathrm{R}$ with the metafor Package. J Stat Softw. 2010;36:1-48.

19. Fernandez-Castillo N, Cabana-Dominguez J, Soriano J, Sanchez-Mora C, Roncero C, Grau-Lopez L, et al. Transcriptomic and genetic studies identify NFAT5 as a candidate gene for cocaine dependence. Transl Psychiatry. 2015;5:1-9.

20. Walker DM, Cates HM, Loh YE, Purushothaman I, Ramakrishnan A, Cahill KM, et al. Cocaine self-administration alters transcriptome-wide responses in the brain's reward circuitry. Biol Psychiatry. 2018;84:867-80.

21. Highfill CA, Baker BM, Stevens SD, Anholt RRH, Mackay TFC. Genetics of cocaine and methamphetamine consumption and preference in Drosophila melanogaster. PLoS Genet. 2019;15:e1007834.

22. Kaun KR, Devineni AV, Heberlein U. Drosophila melanogaster as a model to study drug addiction. Hum Genet. 2012;131:959-75.

23. Wang KH, Penmatsa A, Gouaux E. Neurotransmitter and psychostimulant recognition by the dopamine transporter. Nature. 2015;521:322-7.

24. Mcclung C, Hirsh J. Stereotypic behavioral responses to free-base cocaine and the development of behavioral sensitization in Drosophila. Curr Biol. 1998;8:109-12.

25. Filošević A, Al-samarai S, Andreti R. High throughput measurement of locomotor sensitization to volatilized cocaine in Drosophila melanogaster. Front Mol Neurosci. 2018;11:1-12.

26. de Claro RA, McGinn KM, Verdun N, Lee SL, Chiu HJ, Saber H, et al. FDA Approval: Ibrutinib for patients with previously treated mantle cell lymphoma and previously treated chronic lymphocytic leukemia. Clin Cancer Res. 2015;1:3586-91.

27. Dolgin E. BTK blockers make headway in multiple sclerosis. Nat Biotechnol. 2021;39:3-5.

28. Merolle MI, Ahmed M, Nomie K, Wang ML. The B cell receptor signaling pathway in mantle cell lymphoma. Oncotarget. 2018;9:25332-41.

29. Northcutt AL, Hutchinson MR, Wang X, Baratta MV, Hiranita T, Cochran TA. et al. DAT isn't all that: cocaine reward and reinforcement require Toll-like receptor 4 signaling. Mol Psychiatry. 2015;20:1525-1537.

30. Volkow ND, Fowler JS, Wang GJ, Hitzemann R, Logan J, Schlyer DJ, Dewey SL, Wolf AP. Decreased dopamine D2 receptor availability is associated with reduced frontal metabolism in cocaine abusers. Synapse 1993;14:169-77.

31. Smith RJ, Aston-jones G. Alpha-2 adrenergic and imidazoline receptor agonists prevent cue-induced cocaine-seeking. Biol Psychiatry. 2011;70:712-9.

32. Vandergriff J, Kallman MJ, Rasmussen K. Moxonidine, a selective imidazoline-1 receptor agonist, suppresses the effects of ethanol withdrawal on the acoustic startle response in rats. Biol Psychiatry. 2000;47:874-9.

33. Taksande BG, Kotagale NR, Patel MR, Shelkar GP, Ugale RR, Chopde CT. Agmatine, an endogenous imidazoline receptor ligand modulates ethanol anxiolysis and withdrawal anxiety in rats. Eur J Pharm. 2010;637:89-101.

34. Le Foll B, Di Ciano P, Panlilio LV, Goldberg SR, Ciccocioppo R. Agonists as promising new medications for drug addiction: evidence. Curr Drug Targets. 2013;14:768-76. 
35. Zhong P, Wang W, Yu F, Nazari M, Liu X, Liu Q. Phosphodiesterase 4 inhibition impairs cocaine-induced inhibitory synaptic plasticity and conditioned place preference. Neuropsychopharmacology. 2012;37:2377-87.

36. Olsen CM, Liu Q, Road P. Phosphodiesterase 4 inhibitors and drugs of abuse: current knowledge and therapeutic opportunities. Front Biol. 2017;11:376-86.

37. Satta R, Certa B, He D, Lasek AW. Estrogen receptor $\beta$ in the nucleus accumbens regulates the rewarding properties of cocaine in female mice. Int J Neuropsychopharmacol. 2018;21:382-92.

38. Mccall NM, Kotecki L, Dominguez-lopez S, Marron E. Velasco F De, Carlblom N, et al. Selective ablation of GIRK channels in dopamine neurons alters behavioral effects of cocaine in mice. Neuropsychopharmacology. 2017;42:707-15.

39. Siciliano CA, Jones SR. Cocaine potency at the dopamine rransporter tracks discrete motivational states during cocaine self-administration. Neuropsychopharmacology. 2017;42:1893-904. https://doi.org/10.1038/npp.2017.24.

40. Sadakierska-Chudy A, Frankowska M, Filip M. Mitoepigenetics and drug addiction. Pharmacol Therapeutics. 2014;144:226-33. 1

41. Paydas S. Management of adverse effects/toxicity of ibrutinib. Crit Rev Oncol/ Hematol. 2019;136:56-63.

\section{ACKNOWLEDGEMENTS}

The authors acknowledge the National Institute on Drug Abuse award DP1DA042103 (awarded to RHCP) and DA041613 (awarded to RRHA and TFCM). S.B.H., J.S.H., J.D.W. J.W.W., and J.E.M. have no financial disclosures to declare. We are indebted to the LINCs database curators as well as those responsible for the availability of the RNAsequencing and microarray data. Content is solely the responsibility of the authors and does not necessarily represent the official views of the National Institutes of Health or the Department of Veteran Affairs.

\section{AUTHOR CONTRIBUTIONS}

S.B.H. was the principal analyst, responsible for the study design, data preparation, and data curation as well as writing the initial manuscript. JSH and JDW performed behavioral experiments in Drosophila melanogaster and analyzed these data. J.E.M., J. W.W., T.F.C.M., R.R.H.A., R.H.C.P. were instrumental in reviewing the manuscript, providing expert consultation, and aiding in the interpretation of the findings.

\section{COMPETING INTERESTS}

The authors declare no competing interests.

\section{ADDITIONAL INFORMATION}

Supplementary information The online version contains supplementary material available at https://doi.org/10.1038/s41398-021-01737-5.

Correspondence and requests for materials should be addressed to Spencer B. Huggett or Rohan H. C. Palmer.

Reprints and permission information is available at http://www.nature.com/ reprints

Publisher's note Springer Nature remains neutral with regard to jurisdictional claims in published maps and institutional affiliations. (c) (i) Open Access This article is licensed under a Creative Commons Ay Attribution 4.0 International License, which permits use, sharing, adaptation, distribution and reproduction in any medium or format, as long as you give appropriate credit to the original author(s) and the source, provide a link to the Creative Commons license, and indicate if changes were made. The images or other third party material in this article are included in the article's Creative Commons license, unless indicated otherwise in a credit line to the material. If material is not included in the article's Creative Commons license and your intended use is not permitted by statutory regulation or exceeds the permitted use, you will need to obtain permission directly from the copyright holder. To view a copy of this license, visit http://creativecommons. org/licenses/by/4.0/.

This is a U.S. government work and not under copyright protection in the U.S.; foreign copyright protection may apply 2021 